\title{
Polymerization of C-terminally truncated Mycobacterium tuberculosis FtsZ is unlikely to be physiologically relevant
}

Mycobacterium tuberculosis FtsZ (MtbFtsZ) exhibits unusually slow polymerization kinetics in vitro (White et al., 2000). Recently, Anand et al. (2004) hypothesized that removal of the non-conserved $\mathrm{C}$ terminus would bestow rapid polymerization to MtbFtsZ, comparable to the much faster-polymerizing Escherichia coli FtsZ (EcFtsZ) (Mukherjee \& Lutkenhaus, 1999). Indeed, $\operatorname{MtbFtsZ}(\Delta \mathrm{C} 169)$, in which the last 169 residues were deleted ( $\Delta 211-379$; Fig. 1$)$, was found to polymerize much faster than wild-type MtbFtsZ (Anand et al., 2004). It also formed polymers that were 1.5-times thicker and that exhibited an altered morphology, compared with wild-type MtbFtsZ protofilaments (White et al., 2000). We show here, however, that the three-dimensional structure of $\operatorname{MtbFtsZ}(\Delta \mathrm{C} 169)$ is so perturbed - the truncation exposes a large hydrophobic patch that is normally buried (Fig. 2) that polymerization of MtbFtsZ $(\Delta \mathrm{C} 169)$ is unlikely to be physiologically relevant. The cause(s) of the slow polymerization of wild-type MtbFtsZ must therefore be sought elsewhere.

Three FtsZ crystal structures have been determined: Methanococcus jannaschii FtsZ (MjFtsZ) (Lowe \& Amos, 1998),

Pseudomonas aeruginosa FtsZ (PaFtsZ; complexed to the inhibitor SulA) (Cordell et al., 2003) and, most recently, MtbFtsZ (Leung et al., 2004). FtsZ exhibits significant structural (Nogales et al., 1998b) and functional (Addinall \& Holland, 2002) homology to eukaryotic tubulins. Like tubulin, FtsZs comprise three regions (Fig. 1), the first two of which are conserved (Fig. 3). The N-terminal or GTPase domain (MtbFtsZ residues 1-217) contains the active site, which is encircled by seven conserved 'tubulin' $\mathrm{T}$ loops that supply key guanine nucleotide binding and catalytic (GTP hydrolysis) residues. In particular, 


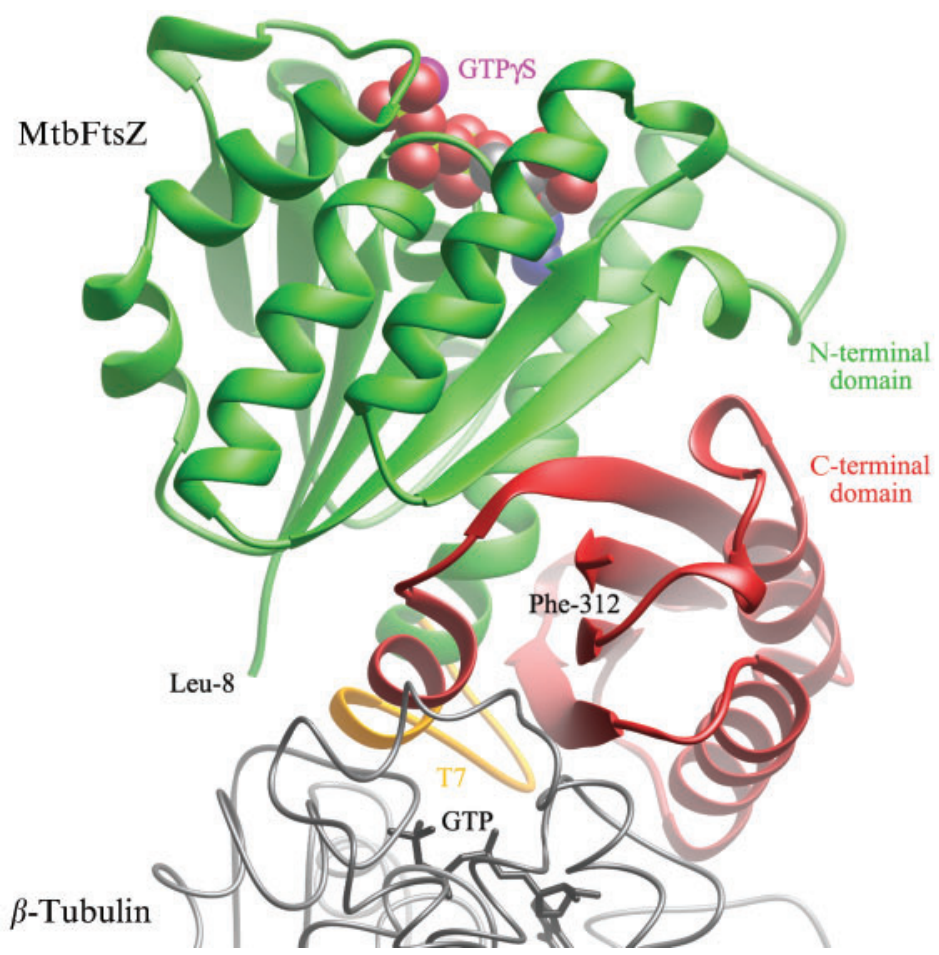

Fig. 1. Fts $Z$ has a tight, two-domain structure. The $\mathrm{N}$ - and C-terminal domains of MtbFtsZ (residues 8-312, GTP $\gamma$ S complex; PDB entry 1RLU, subunit A) are coloured green and red; loop T7 (201-210) is gold. In MtbFtsZ( $\Delta$ C169), N-terminal residues 211-217 (red), the entire C-terminal domain, and the 67 disordered C-terminal tail residues (not shown) are deleted. $\beta$-Tubulin (black), positioned as in a microtubule (the longitudinal protofilament axis is vertical), illustrates both the significant interactions between the C-terminal domain of one FtsZ subunit and the next subunit in the polymer, and how loop T7 completes the active site by binding GTP.

loop T7 (residues 201-210) is supplied in trans to complete the active site as FtsZ subunits polymerize along the longitudinal (protofilament) direction, as demonstrated by mutagenesis (Lu et al., 2001; Scheffers et al., 2002; Wang et al., 1997), and as visualized in the EM structure of tubulin (Fig. 1) (Nogales et al., 1998a). The C-terminal domain (218-312) packs against the $\mathrm{N}$-terminal domain by burying a hydrophobic interface that is contiguous with the hydrophobic cores of the two separate domains (Fig. 2). Both the $\mathrm{N}$ - and C-terminal domains are structurally homologous to the corresponding segments in tubulin; this homology implies that both these domains make subunit-subunit interactions in FtsZ polymers, as they do in microtubules ( $\mathrm{Li}$ et al., 2002; Lowe et al., 2001; Nogales et al., 1998a). In contrast, the C-terminal tail region (313-379) is mostly not well-conserved between different FtsZs
(Fig. 3). This region, which is mostly or completely disordered in all FtsZ crystal structures (Leung et al., 2004), appears to adopt a variety of extended conformations that can interact with other proteins such as ZipA (Mosyak et al., 2000) and FtsW (Datta et al., 2002) to form the septation assembly.

The two ordered FtsZ domains are structurally well-conserved, as is their interaction with one another. For example, the $\mathrm{N}$ - and C-terminal domains of MtbFtsZ and PaFtsZ/MjFtsZ align with r.m.s. deviations of $1 \cdot 0 / 1 \cdot 2 \AA\left(210 / 209 \mathrm{C}^{\alpha}\right.$ atoms $)$ and $1 \cdot 2 / 1 \cdot 3 \AA\left(92 \mathrm{C}^{\alpha}\right)$, respectively; most of the differences are due to mobile surface loops. Together the two domains align with an overall r.m.s. deviation of $1 \cdot 2 / 1 \cdot 4 \AA$ $\left(299 / 300 C^{\alpha}\right)$, demonstrating that the relative disposition of the two domains is also well-conserved.

Removal of MtbFtsZ residues 211-379 therefore removes both the conserved
C-terminal domain and the non-conserved C-terminal tail (Fig. 3). Whereas a tail truncation mutant [i.e. MtbFtsZ( $\Delta$ C68)] may serve as an appropriate probe of the slow polymerization characteristics of MtbFtsZ, removal of the C-terminal domain as well changes the overall structure of FtsZ so as to render results obtained with MtbFtsZ $(\Delta \mathrm{C} 169)$ artefactual. Indeed, the polymerization characteristics of MtbFtsZ( $\Delta \mathrm{C} 169)$ are likely non-physiological for three reasons.

First, removal of the C-terminal domain exposes a large hydrophobic patch on the remaining $\mathrm{N}$-terminal domain in $\operatorname{MtbFtsZ}(\Delta \mathrm{C} 169)$, and would therefore be expected, on structural and biophysical grounds, to create a non-physiological protein prone to aggregation. Notably, MtbFtsZ( $\Delta \mathrm{C} 169)$ had to be purified under denaturing conditions and then refolded (Anand et al., 2004), unlike wild-type MtbFtsZ (White et al., 2000). The N- and C-terminal domains are connected by an extensive buried, hydrophobic interface, as illustrated in Fig. 2. Association of these two domains, as in wild-type MtbFtsZ, buries a total of $2382 \AA^{2}$ of solvent-accessible surface area, a value typical for domain-domain interactions (Jones et al., 2000). The interface is composed of $73 \%$ non-polar atoms, and $50 \%$ of the buried surface area comes from the side chains of 32 hydrophobic residues - $10 \mathrm{Ile}, 8 \mathrm{Leu}, 6 \mathrm{Val}, 3 \mathrm{Met}$, 3 Pro and 2 Phe - out of 60 residues in the interface. (These same side chain types contribute just $17 \%$ of the total surface area for the intact protein.) In short, these are not the characteristics of a dissociable (dispensable) domain interface.

Second, removal of the C-terminal domain would destabilize the conformation of the conserved loop T7. Loop T7 is critical to proper GTP hydrolysis and FtsZ polymerization (Lu et al., 2001; Scheffers et al., 2002; Wang et al., 1997). Of the only ten hydrogen bonds that link the $\mathrm{N}$ - and C-terminal domains, seven cluster tightly between the tip of loop T7 and the C-terminal domain. Furthermore, in MtbFtsZ( $\Delta$ C169), N-terminal domain residues 211-217, which form helix H11 and the link to the C-terminal domain, 


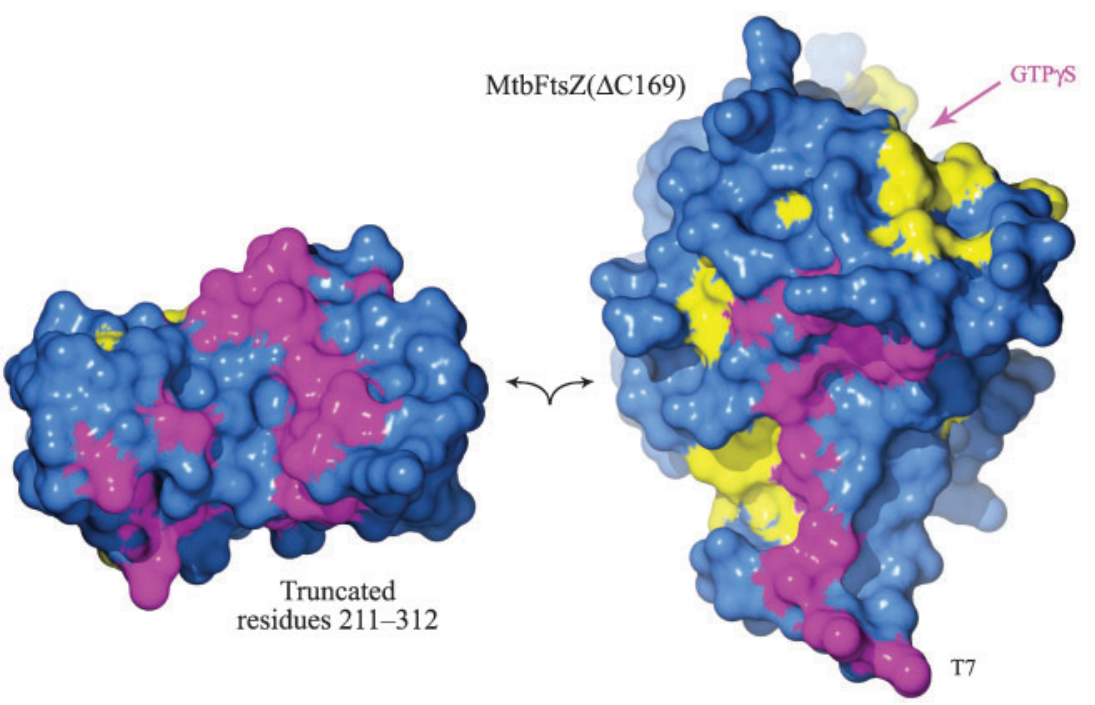

Fig. 2. An extensive hydrophobic interface links the two FtsZ domains. The molecular surface of MtbFtsZ is coloured according to hydrophobicity. The domains of MtbFtsZ are separated and opened like a book (hinge at Ile-214, the centre of the domain interface), with MtbFtsZ( $\Delta C 169)$ on the right and the truncated residues (211-312, i.e. the C-terminal domain) on the left. The surface regions corresponding to the side chain atoms of hydrophobic residues (Ile, Leu, Val, Met, Pro, Phe, Cys, and Tyr) are coloured purple if buried at the $\mathrm{N}$-/C-terminal domain interface, otherwise yellow. The rest of the surface (polar side chains and main chain atoms) is blue. This view is rotated by $\sim 180^{\circ}$ about a vertical axis from that shown in Fig. 1.

are also deleted. It is unclear how the conformation of $\mathrm{T} 7$ can be maintained in the absence of its connections with both the $\mathrm{N}$ - and C-terminal domains.
Third, the tubulin protofilament EM structure (Nogales et al., 1998a) strongly suggests that the C-terminal domain makes substantial intersubunit longitudinal contacts in the FtsZ polymer (Fig. 1). These contacts may not be critical to polymerization, but their loss would likely affect the kinetics of polymerization.

Anand et al. (2004) appear to have omitted several key control experiments. First, how do the polymerization kinetics and polymer morphology of MtbFtsZ( $\Delta$ C169) compare with the analogous EcFtsZ C-terminal deletion mutant? Second, GTP hydrolysis experiments would have differentiated between aggregation and true (proto)filament formation.

Rational mutagenesis of proteins is a powerful tool to help understand their function. With FtsZ, however, it appears that a gentler approach, e.g. $\mathrm{MtbFtsZ}(\Delta \mathrm{C} 68)$, is needed to ensure the physiological relevance of the results obtained.

Figures were generated with Ribbons (Carson, 1991), GRASP (Nicholls et al., 1991), ESPript (Gouet et al., 1999) and Pov4Grasp (Nicolas Calimet, University of Strasbourg, France; http://pov4grasp. free.fr). Surface areas were calculated with GRASP and the Protein-Protein Interaction Server (http://www. biochem.ucl.ac.uk/bsm/PP/server/; Jones et al., 2000).

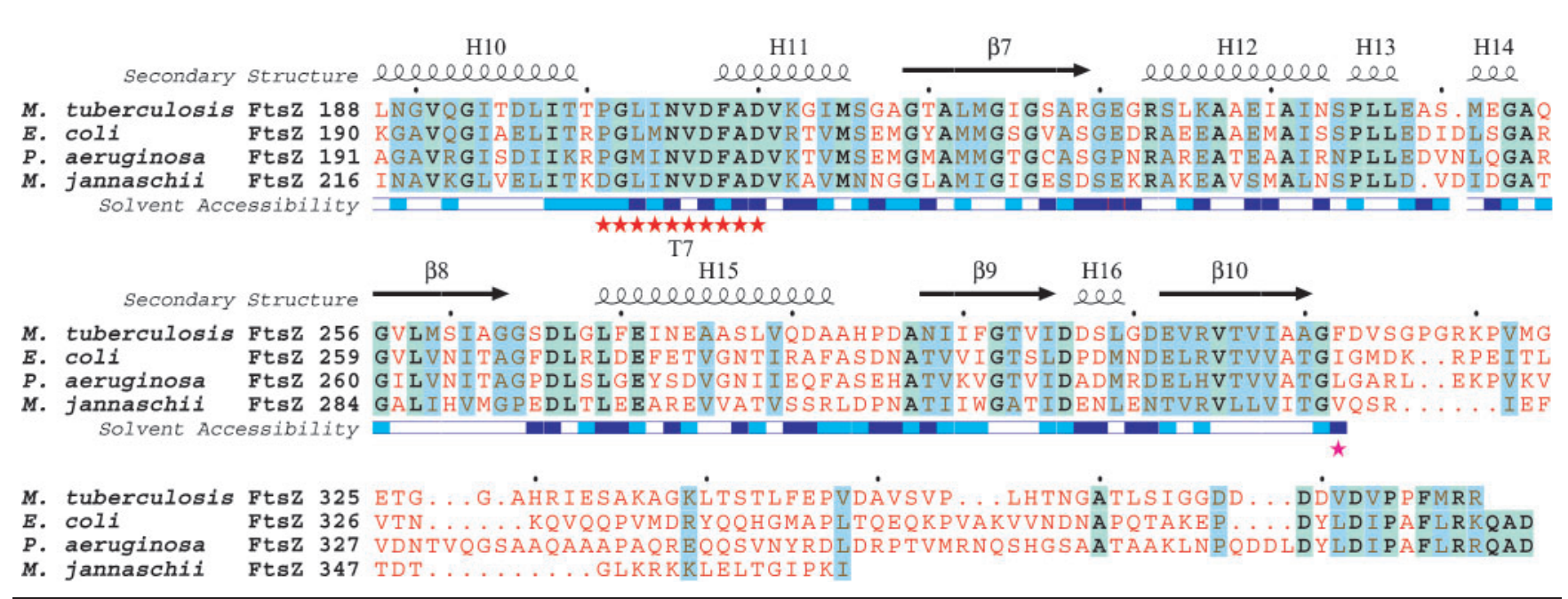

Fig. 3. Most of the residues deleted in MtbFtsZ( $\Delta \mathrm{C} 169)$ are conserved. MtbFtsZ residues 188-379 are aligned with EcFtsZ, PaFtsZ and MjFtsZ. Secondary structure and solvent accessibility (white, buried; blue, accessible) are noted. Identical residues are boxed in green; homologous residues are brown, and non-conserved residues are red. Loop T7 is marked by red stars. The ordered MtbFtsZ structure ends at Phe-312 (purple star), which marks the end of the C-terminal domain. The substantial sequence conservation prior to residue 312 indicates that the three-dimensional structure of EcFtsZ is very similar to the other FtsZs in this region. 


\section{David W. Borhani ${ }^{1}$ and E. Lucile White ${ }^{2}$}

${ }^{1}$ Abbott Bioresearch Center, 100 Research Drive, Worcester, MA 01605 , USA

${ }^{2}$ Drug Discovery Division, Southern Research Institute, Birmingham, AL 35205, USA

Correspondence: David W. Borhani (david.borhani@alum.mit.edu)

Addinall, S. G. \& Holland, B. (2002). The tubulin ancestor, FtsZ, draughtsman, designer and driving force for bacterial cytokinesis. J Mol Biol 318, 219-236.

Anand, S. P., Rajeswari, H., Gupta, P., Srinivasan, R., Indi, S. \& Ajitkumar, P. (2004). A C-terminal deletion mutant of Mycobacterium tuberculosis FtsZ shows fast polymerization in vitro. Microbiology 150, 1119-1121.

Carson, M. (1991). Ribbons 2.0. J Appl Crystallogr 24, 958-961.

Cordell, S. C., Robinson, E. J. \& Lowe, J. (2003). Crystal structure of the SOS cell division inhibitor SulA and in complex with FtsZ. Proc Natl Acad Sci U S A 100, 7889-7894.

Datta, P., Dasgupta, A., Bhakta, S. \& Basu, J. (2002). Interaction between FtsZ and FtsW of Mycobacterium tuberculosis. J Biol Chem 277, 24983-24987.

Gouet, P., Courcelle, E., Stuart, D. I. \& Metoz, F. (1999). ESPript: analysis of multiple sequence alignments in PostScript.

Bioinformatics 15, 305-308.

Jones, S., Marin, A. \& Thornton, J. M. (2000). Protein domain interfaces: characterization and comparison with oligomeric protein interfaces. Protein Eng 13, 77-82.

Leung, A. K. W., White, E. L., Ross, L. J., Reynolds, R. C., DeVito, J. A. \& Borhani, D. W. (2004). Structure of Mycobacterium tuberculosis FtsZ reveals unexpected, G protein-like conformational switches. $J \mathrm{Mol}$ Biol 342, 953-970.

Li, H., DeRosier, D. J., Nicholson, W. V., Nogales, E. \& Downing, K. H. (2002).

Microtubule structure at $8 \AA$ resolution. Structure (Camb) 10, 1317-1328.

Lowe, J. \& Amos, L. A. (1998). Crystal structure of the bacterial cell-division protein FtsZ. Nature 391, 203-206.

Lowe, J., Li, H., Downing, K. H. \& Nogales, E. (2001). Refined structure of alpha beta-tubulin at $3.5 \AA$ resolution. J Mol Biol 313, 1045-1057.

Lu, C., Stricker, J. \& Erickson, H. P. (2001).

Site-specific mutations of FtsZ - effects on GTPase and in vitro assembly. BMC Microbiol $1,7$.

Mosyak, L., Zhang, Y., Glasfeld, E., Haney, S., Stahl, M., Seehra, J. \& Somers, W. S. (2000).
The bacterial cell-division protein ZipA and its interaction with an FtsZ fragment revealed by X-ray crystallography. EMBO J 19, 3179-3191.

Mukherjee, A. \& Lutkenhaus, J. (1999).

Analysis of FtsZ assembly by light scattering and determination of the role of divalent metal cations. J Bacteriol 181, 823-832.

Nicholls, A., Sharp, K. A. \& Honig, B. (1991). Protein folding and association: insights from the interfacial and thermodynamic properties of hydrocarbons. Proteins 11, 281-296.

Nogales, E., Wolf, S. G. \& Downing, K. H. (1998a). Structure of the alpha beta tubulin dimer by electron crystallography. Nature 391, 199-203.

Nogales, E., Downing, K. H., Amos, L. A. \& Lowe, J. (1998b). Tubulin and FtsZ form a distinct family of GTPases. Nat Struct Biol 5, 451-458.

Scheffers, D. J., de Wit, J. G., den Blaauwen, T. \& Driessen, A. J. (2002). GTP hydrolysis of cell division protein FtsZ: evidence that the active site is formed by the association of monomers. Biochemistry 41, 521-529.

Wang, X., Huang, J., Mukherjee, A., Cao, C. \& Lutkenhaus, J. (1997). Analysis of the interaction of FtsZ with itself, GTP, and FtsA. J Bacteriol 179, 5551-5559.

White, E. L., Ross, L. J., Reynolds, R. C., Seitz, L. E., Moore, G. D. \& Borhani, D. W. (2000).

Slow polymerization of Mycobacterium tuberculosis FtsZ. J Bacteriol 182, 4028-4034.

DOI 10.1099/mic.0.27528-0 